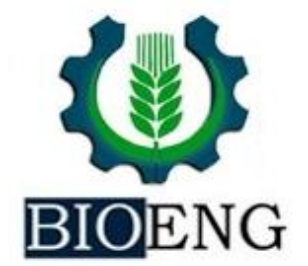

\title{
A INFLUÊNCIA DE DESSECANTE NA QUALIDADE FISIOLÓGICA DE SEMENTES DE SOJA
}

\author{
F. L. Santos ", F. Bertacine, J. S. Souza, I. Simões, J. W. Bossolani, \\ M. E. Sá
}

UNESP - Universidade Estadual Paulista, FEIS, Campus de Ilha Solteira, SP, Brasil

Article history: Received 26 January 2018; Received in revised form 07 March 2018; Accepted 12 March 2018; Available online 27 March 2018.

\section{RESUMO}

O Brasil é o segundo maior produtor de soja no mundo, onde cada vez mais tem-se exigido maiores quantidades e qualidade desta leguminosa. A aplicação de dessecantes que permite a antecipação da colheita em áreas de produção de sementes, tem sido utilizado para minimizar perdas de qualidade. Sedo assim, o objetivo deste trabalho foi analisar a influência da aplicação de dessecante em campos de produção de sementes de soja. O experimento foi conduzido em Delineamento Experimental Inteiramente Casualizado (DIC) disposto em esquema fatorial $(4 \times 2)$ com 4 repetições. Os tratamentos foram constituídos por quatro cultivares de soja: CD 2728 IPRO, SYN 1366C IPRO, BRS 7380 IPRO, BRS 7470 RR com e sem a aplicação de dessecante. Foram realizados os testes de germinação, envelhecimento acelerado, índice de velocidade de germinação, condutividade elétrica, classificação de vigor de plântulas, infestação de fungos na germinação e massa seca de plântulas. A utilização do dessecante influenciou negativamente a qualidade fisiológica de sementes de soja, com exceção do cultivar BRS 7380 RR.

Palavras-chave: Glycyne max, cultivares, herbicida.

\section{INFLUENCE OF THE APPLICATION OF DESICCATION IN THE PHYSIOLOGICAL QUALITY OF SOYBEAN SEEDS}

\begin{abstract}
ABSTRATC
Brazil is the second largest producer of soybeans in the world, where more and more quantities and quality of this legume has been demanded. The application of desiccants that allows anticipation of harvesting in seed production areas has been used to minimize quality losses. Therefore, the objective of this work was to analyze different cultivars of soybean in influence of the application or not of desiccant in function of the physiological quality of the seeds. The experiment was conducted in the Laboratory of Seed Production and Technology with Experimental Design Completely Randomized (DIC) arranged in a factorial scheme (4x2) with 4 replicates. The treatments consisted of four soybean cultivars: CD 2728 IPRO, SYN 1366C IPRO, BRS 7380 IPRO, BRS 7470 RR with and without desiccant application. Tests of germination, accelerated aging, germination speed index, electric conductivity, vigor classification of seedlings, fungal infestation on germination and dry mass of seedlings were performed. The use of desiccant negatively influenced the physiological quality of soybean seeds, with the exception of cultivar BRS 7380 RR.
\end{abstract}

Keywords: Glycyne max, genotypes, herbicide.

\footnotetext{
*fabianalopesagr@gmail.com
} 


\section{INTRODUÇÃO}

A soja (Glycine $\max$ L.) é a leguminosa mais cultivada no mundo, sendo o Brasil o segundo maior produtor. A área de produção no Brasil tem sido protagonista no aumento da produção de soja nos últimos 20 anos, onde o crescimento da área de produção, pode atingir 35,2 milhões de hectares, na safra 2017/18 (CONAB, 2017). Desta forma, a demanda por esta semente também tem crescido ocasionando grandes desafios para produção de sementes de soja, visto que, nas regiões de climas tropicais e subtropicais a deterioração da semente ocorre de forma mais rápida, quando comparada a climas mais frios. (EMBRAPA, 2015).

A utilização de sementes sem qualidade ocasiona os principais problemas para a produção das mesmas, que vai desde a germinação até a colheita, podendo ainda ser afetado por fatores abióticos e bióticos que influenciam a germinação destas. Quando a semente apresenta alta qualidade é devido aos atributos fisiológicos e sanitários (ROCHA et al., 2017), que atuam diretamente na qualidade da semente desde o processo de produção até o armazenamento, tendo uma relação direta no final da cultura em uniformidade, vigor das plantas e produtividade (JUNIOR et al., 2015).

O vigor é responsável pelo alto desempenho que as plantas irão apresentar durante toda sua permanência de desenvolvimento vegetativo e reprodutivo. Para França et al. (2016) como resultado deste vigor obtêm-se a germinação de plântulas com maiores uniformidades e padrões de crescimento, logo, estas plantas, alcançarão profundidades maiores no solo devido ao sistema radicular mais desenvolvido e por fim maiores números de vagens e grãos. A variabilidade genética

\section{MATERIAL E MÉTODOS}

O experimento foi conduzido no Laboratório de Análises de Sementes, pertencente à Faculdade de é responsável por assegurar parte do desenvolvimento da planta, visto que toda a expressão fenotípica é responsável pela genotípica.

Além destes, o clima também afeta diretamente as sementes de soja, já que são altamente higroscópicas, absorvem facilmente a água do ambiente, tendo seu grau de umidade dependente das oscilações do meio. Sendo assim, as sementes sofrem lesões causadas pelas expansões e contrações do tegumento, após uma série de ciclos de umedecimento e secagem, acentuam a sua fragilidade, reduzindo a proteção à semente e causando prejuízos ao seu desempenho (DALTRO et al., 2010).

Para minimizar as alterações que a semente sofre no período final da colheita e garantir o vigor, a utilização de dessecantes permite a antecipação da colheita em áreas de produção de sementes, sendo alternativa de minimizar perdas de qualidade das sementes (EVANGELISTA, 2009).

A utilização da dessecação de sementes é utilizada para diminuir as perdas de qualidade das sementes, tendo em vista que, a eficiência na aplicação visa reduzir perdas de umidade, antecipação da colheita, diminuição de impurezas, para obter sementes de melhor qualidade evitando ao ataque de fungos e pragas no final de ciclo (BEZERRA et al., 2014). A aplicação em cobertura pode não ser eficaz, podendo ocasionar falhas devido a deposição insuficiente de herbicidas e alguns desses dessecantes podem deixar resíduos, proporcionando perda no vigor das sementes (BOTELHO et al., 2016).

Nesse sentido, o objetivo deste trabalho foi analisar a influência da aplicação de dessecante em campos de produção de sementes de soja.

Engenharia/UNESP - Campus de Ilha Solteira, São Paulo.

O Delineamento Experimental utilizado foi o Inteiramente casualizado 
(DIC), disposto em esquema fatorial $(4 \times 2)$ com 4 repetições. Os tratamentos foram constituídos por quatro cultivares de soja, sendo eles CD 2728 IPRO, SYN 1366C IPRO, BRS 7380 IPRO, BRS 7470 RR, com e sem a aplicação de herbicida dessecante quando as mesmas atingiam o estádio de maturidade fisiológica $\left(\mathrm{R}_{7}\right)$. As sementes adquiridas para este experimento foram semeadas na cidade de ParanaíbaMS. Os herbicidas utilizados para dessecação foram paraquat + diquat e óleo mineral, na dosagem recomendada $(2+2+$ $1 \mathrm{~L} \mathrm{ha}^{-1}$, respectivamente).

Para a realização dos testes de qualidade fisiológica de sementes, estas foram previamente tratadas com fipronil,

\section{RESULTADOS E DISCUSSÃO}

Na Tabela 1, podemos observar os valores obtidos para as variáveis primeira contagem (PC), germinação total (GT), índice de velocidade de piraclostrobina e thiophanate methyl. Logo após, foram realizados os testes de germinação, primeira contagem de germinação e germinação total. Além destes, foi efetuado também o teste de envelhecimento acelerado, índice de velocidade de germinação, classificação de vigor de plântulas, infestação de fungos na germinação e massa seca de plântulas. Os resultados foram submetidos ao teste de normalidade de Shapiro-Wilk, e em seguida à análise de variância individual ANOVA pelo teste $F(p \leq 0,05)$, e quando houve diferença significativa, as médias foram comparadas pelo teste de Scott Knott $(\mathrm{p} \leq 0,05)$ para cultivares.

germinação (IVG), envelhecimento acelerado (E.A) e condutividade elétrica (CE).

Tabela 1. Médias observada para primeira contagem (PC), germinação total (GT), índice de velocidade de germinação (IVG), envelhecimento acelerado (E.A) e condutividade elétrica (CE). Ilha Solteira-SP, 2017.

\begin{tabular}{|c|c|c|c|c|}
\hline Tratamentos & $\begin{array}{c}\mathrm{PC} \\
\%\end{array}$ & $\begin{array}{c}\text { GT } \\
\%\end{array}$ & IVG & $\begin{array}{c}\text { E.A } \\
\%\end{array}$ \\
\hline \multicolumn{5}{|l|}{ CULTIVARES } \\
\hline CD 2728 IPRO & $75,63 \mathrm{a}$ & $75,63 \mathrm{a}$ & $7,63 \mathrm{a}$ & $5,31 \mathrm{a}$ \\
\hline SYN 1366C IPRO & $36,25 \mathrm{~b}$ & $36,25 \mathrm{~b}$ & $3,62 \mathrm{~b}$ & $2,41 \mathrm{~b}$ \\
\hline BRS 7470 IPRO & $37,70 \mathrm{~b}$ & $37,70 \mathrm{~b}$ & $3,77 \mathrm{~b}$ & $2,65 \mathrm{~b}$ \\
\hline BRS 7380 RR & $63,55 \mathrm{a}$ & $63,55 \mathrm{a}$ & $6,38 \mathrm{a}$ & $0,00 \mathrm{c}$ \\
\hline \multicolumn{5}{|l|}{ DESSECANTE } \\
\hline Sem & 53,04 & 53,04 & 5,38 & 2,65 \\
\hline Com & 53,52 & 53,52 & 5,31 & 2,53 \\
\hline DMS & 7,47 & 7,47 & 0,80 & 0,54 \\
\hline \multicolumn{5}{|l|}{ VALOR P } \\
\hline CULTIVARES (C) & $0,00 * *$ & $0,00 * *$ & $0,00 * *$ & $0,00 * *$ \\
\hline DESSECANTE (D) & $0,89 \mathrm{~ns}$ & $0,89 \mathrm{~ns}$ & $0,85 \mathrm{~ns}$ & $0,62 \mathrm{~ns}$ \\
\hline $\mathrm{C} \times \mathrm{D}$ & $0,00 * *$ & $0,00 * *$ & $0,00 * *$ & $0,00 * *$ \\
\hline Média & 53,28 & 58,28 & 5,35 & 2,59 \\
\hline C.V. \% & 16,14 & 16,14 & 17,27 & 22,79 \\
\hline
\end{tabular}

Médias seguidas de letra iguais, na coluna, não diferem entre si pelo teste de Scott Knott, a $5 \%$ de probabilidade.

** significativo $\mathrm{p}<0,01 *$ significativo $0,01<\mathrm{p}<0,05 \mathrm{~ns}$ : não significativo.

Os valores obtidos para PC e GT são iguais, uma vez que na segunda contagem (representada pela GT) não houve alteração os valores encontrados 
para a PC, implicando que os cultivares de soja utilizados não apresentam dormência de sementes. Para estes parâmetros, ocorreram interações significativas entre os fatores causais cultivares $\mathrm{x}$ dessecante (Tabela 2). No desdobramento de dessecante dentro de cada cultivar a ausência de aplicação do dessecante proporcionou maiores valores de germinação para todos os cultivares, com exceção do BRS 7380 $\mathrm{RR}$, o qual respondeu de forma negativa a ausência da aplicação, obtendo uma menor germinação, sendo esta cerca de 20\%. De maneira geral, nenhum dos cultivares obtiveram valores de germinação considerados ótimos para comercialização, levando em consideração a recomendação da Regra de Análise de Sementes (RAS) (BRASIL, 2009). Para a aplicação do dessecante apenas o cultivar BRS 7380 $\mathrm{RR}$ respondeu de forma positiva.

Tabela 2. Desdobrando da interação significativa de cultivares e uso de dessecante para primeira contagem (PC\%) e germinação total (GT\%). Ilha Solteira-SP, 2017.

\begin{tabular}{ccccc}
\hline \multirow{2}{*}{ DESSECANTE } & \multicolumn{4}{c}{ CDLTIVARES } \\
\cline { 2 - 5 } & IPRO 2728 & SYN 1366C & BRS 7470 & BRS 7380 \\
& IPRO & IPRO & RR \\
\hline SEM & $81,67 \mathrm{~A}$ & $52,23 \mathrm{~B}$ & $51,23 \mathrm{~B}$ & $29,96 \mathrm{C}$ \\
COM & $69,60 \mathrm{~B}$ & $21,26 \mathrm{C}$ & $24,16 \mathrm{C}$ & $97,13 \mathrm{~A}$ \\
\hline DMS & \multicolumn{5}{c}{14,95} \\
\hline
\end{tabular}

Médias seguidas de mesma letra, maiúscula na linha, não diferem estatisticamente entre si pelo teste de Scott-Knott, a 5\% de probabilidade.

A aplicação do dessecante nos cultivares teve o intuito de evitar a exposição prolongada a fatores bióticos e abióticos que podem interferir na qualidade da semente (BEZERRA et al., 2014). Isto posto, os cultivares CD 2728 IPRO, SYN 1366C IPRO e BRS 7470 IPRO não se beneficiaram desta medida, visto que os herbicidas utilizados (Paraquat e Diquat) agem sobre o fotossistema I, os íons destes herbicidas entram em contato com os elétrons livres do fotossitema produzindo um radical livre que é rapidamente transformado pelo oxigênio em um superperóxido altamente reativo que desidrata as membranas, desta forma pode-se ter ocorrido uma secagem excessiva destas, proporcionando uma suscetibilidade de injurias mecânicas, causando prejuízos para a qualidade fisiológica destas sementes (MARTINS, 2013).

Para o cultivar BRS 7380 RR a medida preventiva contra os fatores bióticos e abióticos (aplicação de dessecante) obteve sucesso, visto que seu período em campo foi menor, mostrando ser um cultivar mais sensível, onde o uso desta aplicação mostrou-se benéfico.

Observa-se na Tabela 3 o IVG, este mesmo indica que quanto mais rápido a velocidade também será a germinação, evitando assim a exposição contra fatores que possam interferir no desenvolvimento da semente. Sendo assim, quando houve a aplicação do dessecante o genótipo BRS 7380 RR foi o que germinou mais rápido, já quando não houve a presença de dessecante $o$ cultivar CD 2728 IPRO obteve o maior IVG. 
Tabela 3. Desdobrando da interação significativa de cultivares e uso de dessecante para o índice de velocidade de germinação (IVG). Ilha Solteira-SP, 2017.

\begin{tabular}{ccccc}
\hline \multirow{2}{*}{ DESSECANTE } & \multicolumn{4}{c}{ CULTIVARES } \\
\cline { 2 - 5 } & $\begin{array}{c}\text { CD 2728 } \\
\text { IPRO }\end{array}$ & $\begin{array}{c}\text { SYN 1366C } \\
\text { IPRO }\end{array}$ & $\begin{array}{c}\text { BRS 7470 } \\
\text { IPRO }\end{array}$ & $\begin{array}{c}\text { BRS 7380 } \\
\text { RR }\end{array}$ \\
\hline SEM & $8,31 \mathrm{~A}$ & $5,12 \mathrm{~B}$ & $5,12 \mathrm{~B}$ & $2,99 \mathrm{~B}$ \\
COM & $6,96 \mathrm{~B}$ & $2,12 \mathrm{C}$ & $2,41 \mathrm{C}$ & $9,76 \mathrm{~A}$ \\
\hline DMS & \multicolumn{5}{c}{1,61} \\
\hline
\end{tabular}

Médias seguidas de mesma letra, maiúscula na linha, não diferem estatisticamente entre si pelo teste de Scott-Knott, a 5\% de probabilidade.

Com relação ao envelhecimento acelerado (EA) (Tabela 4), o CD 2728 IPRO foi o cultivar que obteve um desempenho melhor com relação aos demais cultivares, visto que, este teve as médias mais altas de germinação quando se fez a aplicação ou não do dessecante. Já o cultivar BRS 7470 IPRO foi melhor quando não houve aplicação. No entanto, estes valores não estão dentro dos adequados, segundo os parâmetros da RAS (BRASIL,2009).

Tabela 4: Desdobrando da interação significativa de cultivares e uso de dessecante para envelhecimento acelerado (EA).

\begin{tabular}{ccccc}
\hline & \multicolumn{4}{c}{ CULTIVARES } \\
\cline { 2 - 5 } DESSECANTE & CD 2728 & SYN 1366 & BRS 7470 & BRS 7380 \\
& IPRO & IPRO & IPRO & RR \\
\hline SEM & $5,32 \mathrm{~A}$ & $0 \mathrm{~B}$ & $5,31 \mathrm{~A}$ & $0 \mathrm{~B}$ \\
COM & $5,31 \mathrm{~A}$ & $4,83 \mathrm{~A}$ & $0 \mathrm{~B}$ & $0 \mathrm{~B}$ \\
\hline
\end{tabular}

Médias seguidas de mesma letra, maiúscula na linha, não diferem estatisticamente entre si pelo teste de Scott-Knott, a 5\% de probabilidade.

O teste de EA é o teste mais sensível e eficiente para avaliar o vigor e a resistência ao estresse das sementes, pois as sementes são expostas a condições adversas para seu desenvolvimento, como temperatura e umidade relativa altos. Sementes de menor qualidade quando expostas a estas condições deterioram-se mais rapidamente, interferindo assim, em uma menor taxa de germinação após o EA (SILVA et al., 2016).

Neste teste as sementes absorvem a água que ela é exposta, de modo que os resultados obtidos no mesmo, podem ser influenciados pelo genótipo. Desta forma, as sementes que foram utilizadas neste trabalho, apresentaram valores abaixo dos considerados adequados, o que podemos presumir que estes são mais sensíveis a temperaturas e umidade elevadas.

$\mathrm{Na}$ Tabela 5 podemos observar os resultados obtidos para o vigor de plântulas, sendo constituído de alto, médio e baixo, massa seca de plântula (MSP) e do número de infestação de patógenos nas sementes (INFS). 
Tabela 5. Médias observada para vigor alto, médio e baixo, massa seca de plântula (MSP) e número de infestação nas sementes (INFS). Ilha Solteira-SP, 2017.

\begin{tabular}{|c|c|c|c|c|c|}
\hline \multirow{3}{*}{ Tratamentos } & \multicolumn{3}{|c|}{ VIGOR } & \multirow{3}{*}{$\begin{array}{c}\text { MSP } \\
\mathrm{g}\end{array}$} & \multirow{3}{*}{$\begin{array}{c}\text { INFS } \\
\%\end{array}$} \\
\hline & Alto & Médio & Baixo & & \\
\hline & $\%$ & $\%$ & $\%$ & & \\
\hline \multicolumn{6}{|l|}{ CULTIVARES } \\
\hline CD 2728 IPRO & $12,08 \mathrm{a}$ & $29,96 \mathrm{a}$ & $29,48 \mathrm{ab}$ & $0,28 \mathrm{a}$ & $18,36 \mathrm{a}$ \\
\hline SYN 1366C IPRO & $3,86 \mathrm{~b}$ & $13,05 \mathrm{~b}$ & $19,33 \mathrm{ab}$ & $0,16 \mathrm{~b}$ & 22,23 \\
\hline BRS 7470 IPRO & $7,25 \mathrm{ab}$ & $14,01 \mathrm{~b}$ & $16,43 \mathrm{~b}$ & $0,16 \mathrm{~b}$ & 16,91 \\
\hline BRS 7380 RR & $9,18 \mathrm{ab}$ & $18,85 \mathrm{~b}$ & $35,28 \mathrm{a}$ & $0,25 \mathrm{a}$ & 13,05 \\
\hline \multicolumn{6}{|l|}{ DESSECANTE } \\
\hline Sem & $10,15 \mathrm{a}$ & 19,33 & 24,16 & $0,16 \mathrm{a}$ & 18,36 \\
\hline Com & $6,04 \mathrm{~b}$ & 18,60 & 26,10 & $0,13 \mathrm{~b}$ & 16,91 \\
\hline DMS & 2,87 & 5,32 & 8,89 & 0,03 & 0,03 \\
\hline \multicolumn{6}{|l|}{ VALOR P } \\
\hline CULTIVARES (C) & $0,00 * *$ & $0,00 * *$ & $0,02 *$ & $0,00 * *$ & $0,12 \mathrm{~ns}$ \\
\hline DESSECANTE (D) & $0,00 * *$ & $0,76 \mathrm{~ns}$ & $0,65 \mathrm{~ns}$ & $0,02^{*}$ & $0,57 \mathrm{~ns}$ \\
\hline $\mathrm{C} \times \mathrm{D}$ & $0,00 * *$ & $0,00 * *$ & $0,03 *$ & $0,00 * *$ & $0,00 * *$ \\
\hline Média & 8,09 & 18,97 & 25,13 & 0,21 & 17,64 \\
\hline C.V.\% & 37,99 & 31,05 & 41,13 & 18,45 & 35,19 \\
\hline
\end{tabular}

Médias seguidas de letra iguais, na coluna, não diferem entre si pelo teste de Scott Knott, a 5\% de probabilidade.

$* *$ significativo $\mathrm{p}<0,01 *$ significativo $0,01<\mathrm{p}<0,05 \mathrm{~ns}$ : não significativo.

O cultivar CD 2728 IPRO foi o que obteve maior quantidade de plântulas de alto, médio e baixo vigor, quando não ocorreu a aplicação, como pode ser observado na Tabela 6. Pode-se atribuir que a ausência do dessecante, fez com que as membranas celulares fossem mais bem formadas, diminuindo assim, a liberação de substâncias que são essenciais para o desenvolvimento afetando o vigor das plântulas, logo, estas ficam mais robustas e com maior poder germinativo. 
Tabela 6. Desdobrando da interação significativa de cultivares e uso de dessecante para vigor alto, médio e baixo (\%). Ilha Solteira-SP, 2017.

\begin{tabular}{ccccc}
\hline \multirow{5}{*}{ DESSECANTE } & CD 2728 & SYN 1366C & BRS 7470 & BRS 7380 \\
& IPRO & IPRO & IPRO & RR \\
\cline { 2 - 5 } & \multicolumn{5}{c}{ VIGOR ALTO } \\
\hline SEM & $18,36 \mathrm{~A}$ & $7,73 \mathrm{BC}$ & $14,50 \mathrm{AB}$ & $0,00 \mathrm{C}$ \\
COM & $5,80 \mathrm{~B}$ & $0,00 \mathrm{~B}$ & $0,00 \mathrm{~B}$ & $18,36 \mathrm{~A}$ \\
\hline DMS & \multicolumn{5}{c}{5,47} \\
\hline SEM & $31,90 \mathrm{~A}$ & $22,23 \mathrm{AB}$ \\
COM & $28,03 \mathrm{~A}$ & $3,86 \mathrm{~B}$ \\
\hline DMS & \multicolumn{5}{c}{$10,43 \mathrm{BC}$} & $6,76 \mathrm{C}$ \\
\hline \multicolumn{5}{c}{ VIGOR BAIXO } \\
\hline SEM & $32,86 \mathrm{~A}$ & $21,26 \mathrm{~A}$ \\
COM & $26,10 \mathrm{AB}$ & $17,40 \mathrm{~B}$ \\
\hline DMS & \multicolumn{5}{c}{17,78} \\
\hline
\end{tabular}

Médias seguidas de mesma letra, maiúscula na linha, não diferem estatisticamente entre si pelo teste de Scott-Knott, a 5\% de probabilidade.

Em relação a aplicação de dessecante, o BRS 7380 RR foi o que apresentou o maior número de plântulas nos três parâmetros (alto, médio e baixo) (Tabela 6). O efeito do dessecante neste genótipo promove benefícios, visto que, este sendo mais sensível ao ambiente e demais fatores que possam interferir na sua qualidade, a antecipação da colheita faz com que as sementes apresentem maior vigor e uma alta taxa de germinação.

Verifica-se na Tabela 7 os valores obtidos para massa seca de plântulas (MSP), onde o genótipo CD 2728 IPRO foi o que apresentou maior média, destacando-se dos demais cultivares. Estes valores obtidos entram em concordância com o vigor, visto que este mesmo foi o que apresentou maior quantidade de plântulas, isto posto, ocorre uma maior transferência de matéria seca dos tecidos embrionários para o eixo embrionário, ficando assim, uma plântula mais vigorosa e consequentemente com maior quantidade de matéria seca (ISELY,1956).

Tabela 7. Desdobrando da interação significativa de cultivares e uso de dessecante para massa seca de plântula (g). Ilha Solteira-SP, 2017.

\begin{tabular}{ccccc}
\hline \multirow{2}{*}{ DESSECANTE } & CD 2728 & SYN 1366C & BRS 7470 & BRS 7380 \\
& IPRO & IPRO & IPRO & RR \\
\hline SEM & $0,32 \mathrm{~A}$ & $0,24 \mathrm{~B}$ & $0,22 \mathrm{~B}$ & $0,15 \mathrm{~B}$ \\
COM & $0,25 \mathrm{~B}$ & $0,08 \mathrm{~B}$ & $0,10 \mathrm{~B}$ & $0,35 \mathrm{~A}$ \\
\hline DMS & \multicolumn{5}{c}{0,07} \\
\hline
\end{tabular}

Médias seguidas de mesma letra, maiúscula na linha, não diferem estatisticamente entre si pelo teste de Scott-Knott, a 5\% de probabilidade.

A aplicação do dessecante fez com que o BRS 7380 RR fosse melhor, tendo uma maior quantidade de matéria seca, afirmando que para este genótipo o melhor é que se faça a aplicação, 
antecipando a sua colheita para que não ocorra a perda de vigor das sementes.

Nota-se na Tabela 8, que para a quantidade de sementes que sofreram infestação (INFS), o CD 2728 IPRO foi o melhor sem a aplicação do dessecante, ficando este com a menor média, já quando houve a aplicação este apresentou um comportamento contrário, ficando com a maior, dados estes que corroboram com o vigor, pois a aplicação fez com as sementes de menor vigor liberassem mais exsudados propiciando uma maior infestação de semente (DOS SANTOS, 2016).

Tabela 8. Desdobrando da interação significativa de cultivares e uso de dessecante para número de infestação de patógenos nas sementes (INFS). Ilha Solteira-SP, 2017.

\begin{tabular}{ccccc}
\hline \multirow{2}{*}{ DESSECANTE } & \multicolumn{4}{c}{ CULTIVARES } \\
\cline { 2 - 5 } & CD 2728 & SYN 1366C & BRS 7470 & BRS 7380 \\
& IPRO & IPRO & IPRO & RR \\
\hline SEM & $4,83 \mathrm{~B}$ & $36,67 \mathrm{~A}$ & $19,33 \mathrm{~B}$ & $10,63 \mathrm{AB}$ \\
COM & $31,90 \mathrm{~A}$ & $5,80 \mathrm{~B}$ & $14,50 \mathrm{~B}$ & $15,46 \mathrm{~B}$ \\
\hline DMS & \multicolumn{4}{c}{0,07} \\
\hline
\end{tabular}

Médias seguidas de mesma letra, maiúscula na linha, não diferem estatisticamente entre si pelo teste de Scott-Knott, a 5\% de probabilidade.

\section{CONCLUSÃO}

$\mathrm{O}$ vigor de sementes do cultivar CD 2728 IPRO é superior quando não ocorre a aplicação de dessecante, enquanto

\section{REFERÊNCIAS BIBLIOGRÁFICAS}

BEZERRA, A. R. G.; SEDIYAMA, T.; NOBRE, D. A.; FERREIRA, L. V.; SILVA, F.; SILVA, A. F.; ROSA, D. P. Efeito da dessecação com ethefon na produção e qualidade da soja. Viçosa. MG: Revista de Ciências Agrárias, 37(3), 2014.

BOTELHO, E. J. F.; OLIVEIRA, J. A; VON PINHO, É. V. D. R.; CARVALHO, E. R. Qualidade de sementes de soja obtidas de diferentes cultivares submetidas à dessecação com diferentes herbicidas $\mathrm{e}$ épocas de aplicação. Boa Vista. RR: RevistaAgro@mbiente Online, 10 (2), 2016.

BRASIL. Ministério da Agricultura, Pecuária e Abastecimento. Secretária de defesa agropecuária. Regras para análises de sementes, 2009. na presença de dessecante, o genótipo BRS 7380 RR apresenta maior vigor de sementes.

CONAB. Companhia Nacional de Abastecimento. Acompanhamento de safra brasileira: grãos: safra 2017/18, Primeiro levantamento de outubro. Brasília, 2017.

DALTRO, E. M. F.; ALBURQUEQUE, M. D. F.; FRANÇA NETO, J. D. B.; GUIMARÃES, S. C.; GAZZIERO, D. L. P.; HENNING, A. A. Aplicação de dessecantes em pré-colheita: efeito na qualidade fisiológica de sementes de soja. Revista Brasileira de Sementes, 32 (1): 111-122, 2010.

DOS SANTOS, J.; MÜHL, F. R.; MOREIRA, A.; RITTER, A. F. S.; FELDMANN, N. A.; RHODEN, A.; BALBINOT, M. Avaliação da qualidade fisiológica e sanitária de sementes de soja produzidas no município de frederico westphalen/RS. Revista de Ciências Agroveterinárias e Alimentos, n. 1, 2016. 
EMBRAPA. "Semente de soja de qualidade é primeiro passo para sucesso da safra". 2015.

EVANGELISTA, J. R. E. Dessecantes na produção e qualidade de sementes de soja [Glycine max (L) Merrill]. 2009.

FERREIRA, D. F. Sisvar - sistema de análise de variância para dados balanceados. Lavras: UFLA. 19 p., 2011.

FRANÇA-NETO, J. D. B.; KRZYZANOWSKI, F. C.; HENNING, A. A.; DE PÁDUA, G. P.; LORINI, I.; HENNING, F. A. "Tecnologia da produção de semente de soja de alta qualidade". Embrapa Soja-Documentos (INFOTECA-E), 2016.

ISELY, D. OPPORTUNITIES IN SEED TECHNOLOGY (SEED ANALYSIS). In: Proceedings of the Association of Official Seed Analysts. The Association of Official Seed Analysts, 1956. p. 43-45.

JUNIOR, A. Z.; SILVEIRA, D. C.; BONETTI, L. P.; SILVEIRA, R. S.; NETO, O. B. Avaliação da qualidade fisiológica de sementes de culturas oleaginosas em diferentes substratos. Salão do Conhecimento, 1 (1), 2015.

MARCOS-FILHO, J. Fisiologia de Sementes de Plantas Cultivadas. Londrina, PR: Abrantes, 2015. 659. p.

MARTINS, T. Herbicida Paraquat: conceitos, modo de ação e doenças relacionadas. Semina: Ciências Biológicas e da Saúde, 34 (2): 175-186, 2013.

MATHIAS, $\quad$ V.; PEREIRA, T.; MANTOVANI, A., ZÍLIO, M.; MIOTTO, P.; COELHO, C. M. M. Implicações da época de colheita sobre a qualidade fisiológica de sementes de soja. Revista Agro@mbiente On-line, 11 (3): 223-231, 2017.
MEDEIROS, M. A.; COSTA, G. L. Maturação fisiológica de sementes de maxixe (Cucumis anguria L.). Revista Brasileira de Sementes,32 (3): 17-24, 2010.

ROCHA, G. C.; NETO, A. R.; CRUZ, S. J. S.; CAMPOS, G. W. B.; OLIVEIRA, C. A. C.; SIMON, G. A. Qualidade fisiológica de sementes de soja tratadas e armazenadasPhysiological quality of treated and stored soybean_ seeds. Científica@ Multidisciplinary Journal,4 (1): 50-65, 2017.

SILVA, R. S.; VENDRUSCOLO, T. P. S.; CASTRILLON, M. A.; CARLA CORREAA, L. I. M. A.; BARELLI, M. A. A. Qualidade fisiológica de sementes de sorgo biomassa (Sorghum bicolor L. Moench). Revista Espacios, 37 (31), 2016. 